\title{
Gastroesophageal Stents for the Management of Post Sleeve Gastrectomy Leakage: Interventional Radiology Experience
}

\author{
Abdulmajeed Bin Dahmash ${ }^{1} \quad$ Mohammad Arabi $^{1} \quad$ Aljabriyah Alfutais $^{2} \quad$ Mohammed A. Alswayah $^{1}$ \\ Shaker Alshehri ${ }^{1}$ Refaat Salman ${ }^{1} \quad$ Yousof Al Zahrani $^{1} \quad$ Mohammed Almoaiqel $^{1}$
}

\footnotetext{
1 Vascular and Interventional Radiology Unit, Department of Medical Imaging, King Abdulaziz Medical City \& King Abdullah Specialized Children's Hospital, Ministry of National Guard-Health Affairs, Riyadh, Saudi Arabia

2 Vascular and Interventional Radiology Unit, Department of Medical Imaging, King Abdullah Bin Abdulaziz University Hospital, Princess Nourah Bint Abdulrahman University, Riyadh, Saudi Arabia
}

Address for correspondence Mohammad Arabi, MD, Vascular and Interventional Radiology Unit, Department of Medical Imaging, King Abdulaziz Medical City, Ministry of National Guard-Health Affairs, Riyadh 22490-11426, Kingdom of Saudi Arabia (e-mail: marabi2004@hotmail.com).

Arab J Intervent Radiol 2021;5:102-105.

\author{
Abstract \\ Keywords \\ - sleeve gastrectomy \\ leakage \\ - gastric sleeve leak \\ - gastroesophageal \\ stent \\ - minimally invasive \\ - interventional \\ radiology
}

Objective The aim of this study was to assess the efficacy and safety of retrievable, self-expandable gastroesophageal stents to manage gastric leaks post sleeve gastrectomy by interventional radiology (IR).

Methods This is a retrospective study of patients with gastric leaks post sleeve gastrectomy who were managed with gastroesophageal stent placement. The diameters of the stents ranged from 23 to $30 \mathrm{~mm}$ and the lengths 12.5 to $24 \mathrm{~cm}$.

Results Thirty-one patients (mean age, $32.4 \pm 7.97$ years) were included in the study, and 46 stents were placed. The total average stenting time was $59.1 \pm 35.5$ days. Leakage resolved in $93.5 \%$ of the patients. Thirty-eight (82.6\%) of the stents were retrieved by IR, and the remaining stents were retrieved under endoscopic visualization. Stricture occurred as a complication in four patients: three of which were treated with stenting (one by IR and two by endoscopy) and one required esophagoplasty. Six patients experienced stent migration, three of which underwent stent adjustment, while the remaining three underwent stent reinsertion

Conclusion Gastroesophageal stent placement by IR for gastric leaks post sleeve gastrectomy is safe and effective in our study.

\section{Introduction}

Sleeve gastrectomy is gaining popularity among bariatric surgeons due to its efficacy in inducing weight loss as well as its technical simplicity and lower rate of complications compared with other surgical procedures. Currently, sleeve gastrectomy is the most common bariatric surgery globally. ${ }^{1-3}$ Nevertheless, gastric leaks remain a challenging concern due to difficulties in their management and associated patient comorbidities. ${ }^{4-6}$ Several surgical, endoscopic, and published online February 7, 2022
DOI https://doi.org/ $10.1055 / \mathrm{s}-0041-1742086$ ISSN 2542-7075. (c) 2022. The Pan Arab Interventional Radiology Society. All rights reserved.

This is an open access article published by Thieme under the terms of the Creative Commons Attribution-NonDerivative-NonCommercial-License, permitting copying and reproduction so long as the original work is given appropriate credit. Contents may not be used for commercial purposes, or adapted, remixed, transformed or built upon. (https://creativecommons.org/ licenses/by-nc-nd/4.0/)

Thieme Medical and Scientific Publishers Pvt. Ltd., A-12, 2nd Floor, Sector 2, Noida-201301 UP, India 
radiological techniques have been utilized to achieve effective sealing of gastric leaks. ${ }^{7-11}$ The incidence of gastric leaks post bariatric surgeries ranges between 0.1 and $5.6 \%$, and pathogenesis of these leakages is triggered by ischemic or mechanical causes. Several risk factors may predispose postoperative leaks including previous bariatric surgery, body mass index, diabetes, hypertension, and others. ${ }^{8}$

Unlike the endoscopic approach to treat gastric leaks post sleeve gastrectomy, there is scant literature on the treatment of gastric leaks using gastroesophageal stents by interventional radiology (IR). A study by Guzaiz et $\mathrm{al}^{7}$ investigated using gastroesophageal stents by IR in 12 patients with post gastric sleeve leakage and demonstrated leak healing in all patients. Furthermore, a study from Spain by Serra et al ${ }^{12}$ included six patients with gastric leaks who were treated with stents by IR. The intervention was successful in five patients.

The aim of this study is to evaluate the effectiveness and safety of retrievable, self-expandable covered stents by IR to treat gastric leaks post sleeve gastrectomy.

\section{Methods}

\section{Study Design and Setting}

In this retrospective study, all patients who underwent sleeve gastrectomy complicated by leakage and managed with gastroesophageal stenting by IR between July 2014 and December 2019 were included. Leakage was diagnosed by either computed tomography (CT) scan or by an upper gastrointestinal study. The Institutional Review Board approved the study and waived the need for informed consent.

\section{Patients}

Patients who were treated by surgical reintervention and patients with missing data and/or follow-up were excluded from the study; only patients with the Niti-S $S^{\mathrm{T}}$ esophageal stent (Taewoong Medical, Gyeonggi-Do, South Korea) as their initial stent or for restenting after failure of other stent types were included in this study to improve the homogeneity of the cohort characteristics. This particular make of stent was selected because it is the most frequently used type of stent in our unit. Age, sex, date of stent implantation, date of stent retrieval, type of stent, and the stent diameter and length were obtained from patients' electronic chart. The clinical and imaging data obtained for each patient during the follow-up were also included in this study.

\section{Procedure Details}

Stent placement and retrieval were completed under general anesthesia. The presence of the leakage was detected using a water-soluble contrast injection in the distal esophagus (-Fig. 1). The gastroesophageal stent was introduced via transoral approach using a super stiff guidewire without a sheath after cannulating the duodenum ( - Fig. 2). Occasional oral and nasal suctioning were done to lower the risk of aspiration. Nutritional intake either through a nasojejunal tube or via total parenteral nutrition and solid oral intake was resumed as tolerated. Stent retrieval by IR was achieved

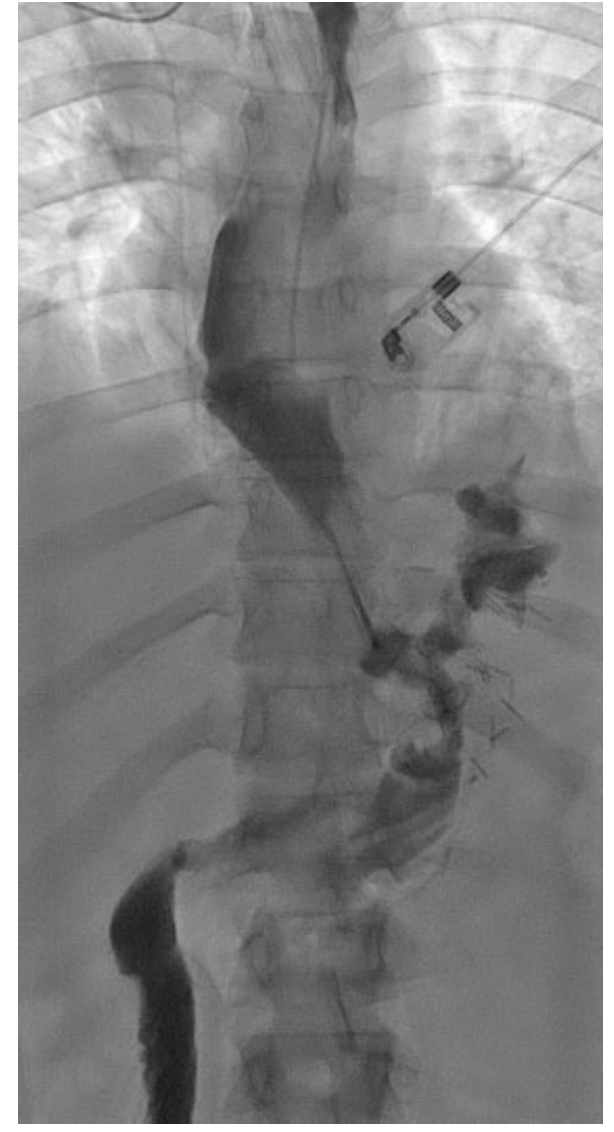

Fig. 1 Intraprocedural fluoroscopy showing staple line leak.

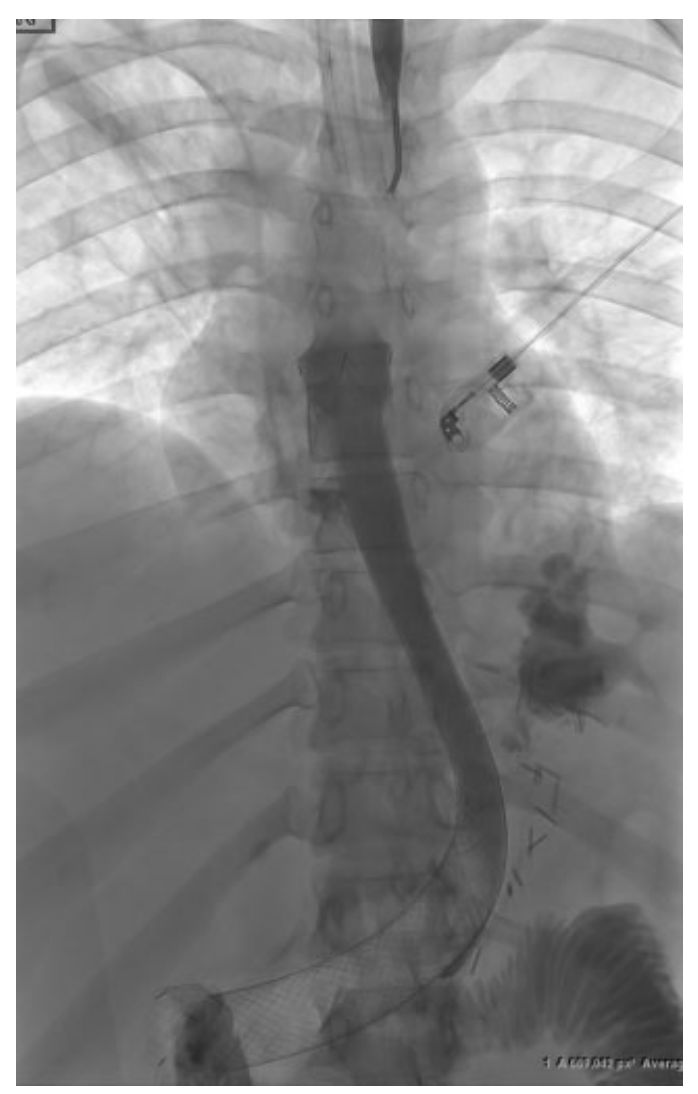

Fig. 2 Fully covered gastroesophageal stent deployed successfully. 


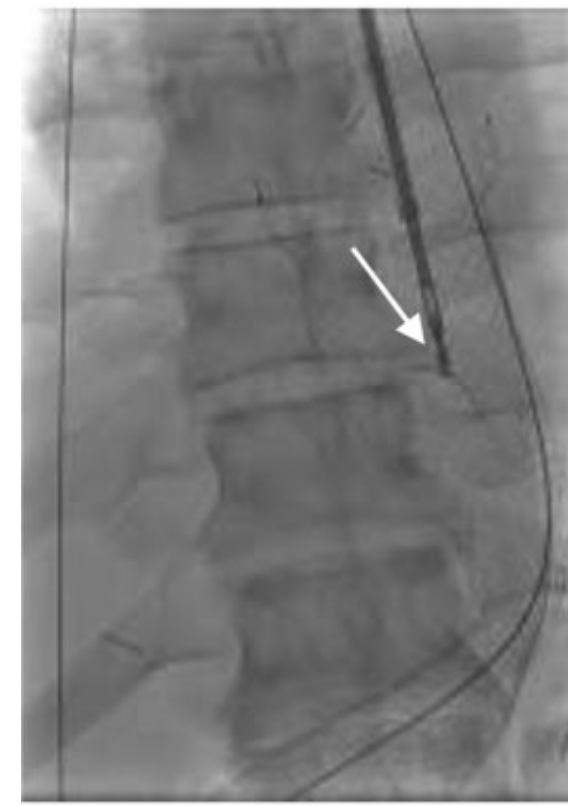

A

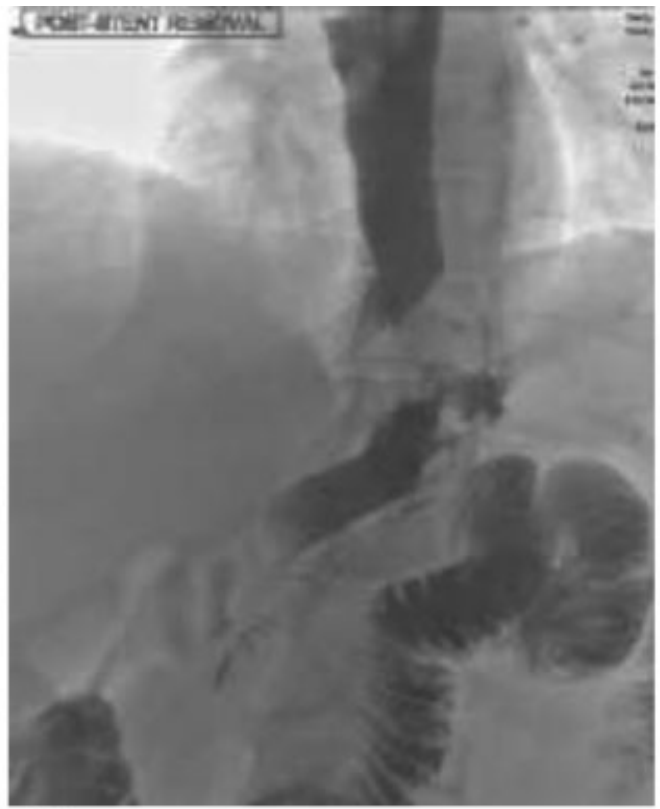

B

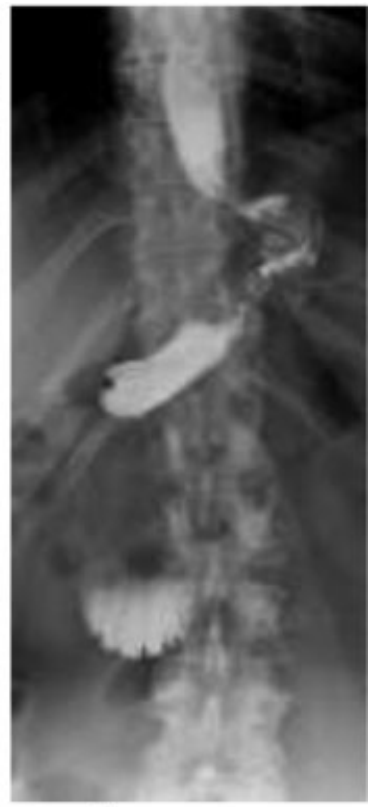

C

Fig. 3 Intraprocedural fluoroscopy image showing (A) forceps (arrow) capturing the stent. (B) Intraprocedural post stent removal image showing no residual leak. (C) Follow-up upper gastrointestinal study after 4 months showing no leak.

by capturing and removing the stent using the Raptor grasping device (US endoscopy, Mentor, Ohio, United States) via an oral approach (-Fig. 3). Post retrieval, an upper gastrointestinal study, and/or CT scan were done to identify persistent leaks. Clinical success was defined as no leak in the final outcome.

\section{Statistical Analysis}

The statistical analysis was performed using $\mathrm{R}$ version 3.6.2. (RStudio, Inc., Boston, Massachusetts, United States). The continuous variables were summarized using mean \pm standard deviation or median (interquartile range), as appropriate, and the categorical variables were summarized using counts and percentages. Binary logistic regression was used to assess the factors associated with stent migration. Hypothesis testing was performed at a $5 \%$ level of significance.

\section{Results}

Thirty-one patients (16 males and 15 females) with a mean age of $32.4 \pm 7.97$ years were included in this study. A total of six patients were excluded from this study. The majority $(89.17 \% ; n=41)$ of the stents used were Niti-S. Four $(8.7 \%)$ Hanarostents (M.I.Tech, Gyeonggi-Do, Korea) and one (2.17\%) WallFlex stent (Boston Scientific, Natick, Massachusetts, United States) were also used. The diameters of the stents ranged from $23 \mathrm{~mm}$ to $30 \mathrm{~mm}$, and the lengths were between $12.5 \mathrm{~cm}$ and $24 \mathrm{~cm}$. The average time of each individual stent placement to retrieval was $38.9 \pm 21$ days. The average total stenting time (from first stent placed to last stent removed for each patient) was $59.1 \pm 35.5$ days.

Reintervention was required in 14 (45.2\%) of the patients. The average time to reintervention was $24.2 \pm 17.6$ days (-Table 1). The main reasons for reintervention included
Table 1 Summary of reinterventions used in the included patients

\begin{tabular}{|l|l|l|}
\hline Reintervention: & & \\
\hline No & $17(54.8 \%)$ & \\
\hline Yes & $14(45.2 \%)$ & \\
\hline Time to reintervention (days) & $24.2 \pm 17.6$ & \\
\hline Stent reinsertion: & & \\
\hline No & $21(67.7 \%)$ & \\
\hline Yes & $10(32.3 \%)$ & \\
\hline Reason for reintervention: & & 14 \\
\hline Leak & $7(22.5 \%)$ & \\
\hline Migration & $6(19.35 \%)$ & \\
\hline Stricture & $1(3.22 \%)$ & \\
\hline Removal mechanism: & & 46 \\
\hline Endoscopy & $8(17.4 \%)$ & \\
\hline IR & $38(82.6 \%)$ & \\
\hline Final outcome: & & 31 \\
\hline Persistent leak & $2(6.45 \%)$ & \\
\hline No leak & $29(93.55 \%)$ & \\
\hline
\end{tabular}

Abbreviation: IR, interventional radiology.

persistent leak $(n=7,22.5 \%)$, stent migration $(n=6,19.35 \%)$, and stricture $(n=1,3.22 \%)$. Stent adjustment was required in three patients due to migration, and the remaining three patients with stent migration underwent stent reinsertion. Leak was controlled in 29 (93.5\%) of the patients. Leak persisted in two patients who underwent reintervention by endoscopy. Eight (17.4\%) stent retrieval procedures 
were performed using endoscopic visualization. The remaining stents $(82.6 \%, n=38)$ were removed by IR. One patient with stricture required esophagoplasty, and two patients with stricture underwent restenting by endoscopy.

\section{Discussion}

This retrospective study explored the effectiveness and complications of using retrievable self-expandable stents to manage gastric leak post sleeve gastrectomy by IR among 31 patients in a tertiary hospital in Saudi Arabia. The success rate (defined as no leak in the final outcome) was $93.55 \%$, which is comparable to the success rate of the endoscopic management reported in a meta-analysis conducted by Puli et $\mathrm{al}^{8}{ }^{8}$ which included 67 patients in seven studies with a success rate of $87.7 \%$. In addition, a study from Kuwait on 17 patients showed a success rate of $76 \%$ using an endoscopic approach, and the median duration of the stenting time was 42 days. ${ }^{13}$ In our study, the average time of each individual stent placement was $38.9 \pm 21$ days and the average total stenting time (from first stent placed to last stent removed) was $59.1 \pm 35.5$ days.

About $55 \%$ of our patients underwent stenting only once and did not require any reintervention (including new stent insertion, stent adjustment, and collection drainage). The causes of reinterventions among the patients in this study were persistent leak (50\%), stent migration (43\%), and stricture (7\%). Similarly, in the study by Klimczak et al, ${ }^{14}$ which included 14 patients and used Niti-S (mega) stents, restenting was required in $46 \%$ of the patients. Moreover, a study in Saudi Arabia showed that $82.8 \%$ of the patients underwent stenting only once and the patients who received partially covered self-expandable metal stents were significantly more likely to undergo stenting only once compared with those who received fully covered stents. ${ }^{15}$

Four (13\%) patients in this study had stricture as a complication. Three were treated by stenting (one managed by IR and two by endoscopy), and one patient underwent esophagoplasty. Similarly, in Murino et al's study, $14 \%$ of the patients had stricture as a complication, $5 \%$ had hemorrhage, and $2 \%$ had perforation. ${ }^{16}$ A systematic review by Hughes et al ${ }^{17}$ included 26 articles on different treatment methods of post sleeve gastrectomy leakage, and it showed $62 \%$ success rate in patients who initially treated endoscopically, $76 \%$ in patient who were managed surgically, and $82 \%$ in patients managed conservatively.

The limitations of this study include its retrospective single-center design, multiple operators, and variable follow-up. Nevertheless, this study demonstrated the safety and effectiveness of the IR approach to treat gastric leaks post sleeve gastrectomy. More studies investigating the IR approach to treat gastric leaks are warranted to determine the effectiveness and complications of the IR method.

\section{Funding}

There is no source of fund for this study.
Conflict of Interest

The authors declare no conflict of interest.

\section{References}

1 Ponce J, DeMaria EJ, Nguyen NT, Hutter M, Sudan R, Morton JM. American Society for Metabolic and Bariatric Surgery estimation of bariatric surgery procedures in 2015 and surgeon workforce in the United States. Surg Obes Relat Dis 2016;12(09):1637-1639

2 Buchwald H, Oien DM. Metabolic/bariatric surgery worldwide 2011. Obes Surg 2013;23(04):427-436

3 English WJ, DeMaria EJ, Brethauer SA, Mattar SG, Rosenthal RJ, Morton JM. American Society for Metabolic and Bariatric Surgery estimation of metabolic and bariatric procedures performed in the United States in 2016. Surg Obes Relat Dis 2018;14(03): 259-263

4 Podnos YD, Jimenez JC, Wilson SE, Stevens CM, Nguyen NT. Complications after laparoscopic gastric bypass: a review of 3464 cases. Arch Surg 2003;138(09):957-961

5 Marshall JS, Srivastava A, Gupta SK, Rossi TR, DeBord JR. Roux-enY gastric bypass leak complications. Arch Surg 2003;138(05): 520-523, discussion 523-524

6 Jurowich C, Thalheimer A, Seyfried F, et al. Gastric leakage after sleeve gastrectomy-clinical presentation and therapeutic options. Langenbecks Arch Surg 2011;396(07):981-987

7 Guzaiz N, Arabi M, Khankan A, et al. Gastroesophageal stenting for the management of post sleeve gastrectomy leak. A single institution experience. Saudi Med J 2016;37(12):1339-1343

8 Puli SR, Spofford IS, Thompson CC. Use of self-expandable stents in the treatment of bariatric surgery leaks: a systematic review and meta-analysis. Gastrointest Endosc 2012;75(02):287-293

9 El Mourad H, Himpens J, Verhofstadt J. Stent treatment for fistula after obesity surgery: results in 47 consecutive patients. Surg Endosc 2013;27(03):808-816

10 Sakran N, Goitein D, Raziel A, et al. Gastric leaks after sleeve gastrectomy: a multicenter experience with 2,834 patients. Surg Endosc 2013;27(01):240-245

11 Bège T, Emungania O, Vitton V, et al. An endoscopic strategy for management of anastomotic complications from bariatric surgery: a prospective study. Gastrointest Endosc 2011;73(02): 238-244

12 Serra C, Baltasar A, Andreo L, et al. Treatment of gastric leaks with coated self-expanding stents after sleeve gastrectomy. Obes Surg 2007;17(07):866-872

13 Alazmi W, Al-Sabah S, Ali DAM, Almazeedi S. Treating sleeve gastrectomy leak with endoscopic stenting: the Kuwaiti experience and review of recent literature. Surg Endosc 2014;28(12): 3425-3428

14 Klimczak T, Klimczak J, Szewczyk T, Janczak P, Jurałowicz P. Endoscopic treatment of leaks after laparoscopic sleeve gastrectomy using MEGA esophageal covered stents. Surg Endosc 2018; 32(04):2038-2045

15 Almadi MA, Bamihriz F, Alharbi O, et al. Use of self-expandable metal stents in the treatment of leaks complicating laparoscopic sleeve gastrectomy: a cohort study. Obes Surg 2018;28(06): 1562-1570

16 Murino A, Arvanitakis M, Le Moine O, Blero D, Devière J, Eisendrath P. Effectiveness of endoscopic management using selfexpandable metal stents in a large cohort of patients with postbariatric leaks. Obes Surg 2015;25(09):1569-1576

17 Hughes D, Hughes I, Khanna A. Management of staple line leaks following sleeve gastrectomy-a systematic review. Obes Surg 2019;29(09):2759-2772 